\title{
BMJ Open Study rationale and protocol of the BARICO study: a longitudinal, prospective, observational study to evaluate the effects of weight loss on brain function and structure after bariatric surgery
}

Debby Vreeken, ${ }^{1,2}$ Maximilian Wiesmann, ${ }^{2}$ Laura N Deden, ${ }^{1}$ Ilse A C Arnoldussen, ${ }^{2}$ Esther Aarts, ${ }^{3}$ Roy P C Kessels, ${ }^{3,4,5}$ Robert Kleemann, ${ }^{6}$ Eric J Hazebroek, ${ }^{1}$ Edo O Aarts, ${ }^{1}$ Amanda J Kiliaan ${ }^{2}$

To cite: Vreeken $\mathrm{D}$, Wiesmann M, Deden LN, et al. Study rationale and protocol of the BARICO study: a Iongitudinal, prospective, observational study to evaluate the effects of weight loss on brain function and structure after bariatric surgery. BMJ Open 2019;9:e025464. doi:10.1136/ bmjopen-2018-025464

- Prepublication history and additional material for this paper are available online. To view these files, please visit the journal online (http://dx.doi. org/10.1136/bmjopen-2018025464).

EOA and AJK contributed equally.

Received 16 July 2018 Revised 23 November 2018 Accepted 28 November 2018

Check for updates

(C) Author(s) (or their employer(s)) 2019. Re-use permitted under CC BY-NC. No commercial re-use. See rights and permissions. Published by BMJ.

For numbered affiliations see end of article.

Correspondence to

Dr Amanda J Kiliaan;

amanda.kiliaan@radboudumc.nl

\section{ABSTRACT}

Introduction Weight loss after bariatric surgery (BS) is often associated with improved cognition and structural brain recovery. However, improved cognition after BS is not always exhibited by patients, in fact, in some cases there is even a decline in cognition. Long-term consequences of BS weight loss, in terms of obesity and related diseases, can be hard to determine due to studies having short follow-up periods and small sample sizes. The aim of the BARICO study (BAriatric surgery Rijnstate and Radboudumc neuro/maging and Cognition in Obesity) is to determine the long-term effect of weight loss after BS on brain function and structure, using sensitive neuropsychological tests and (functional) MRI ((f)MRI). Secondary study endpoints are associated with changes in metabolic and inflammation status of adipose tissue, liver and gut, in relation to brain structure and function. Also, the possible correlation between weight loss, gut microbiota composition change and neuropsychological outcomes will be investigated.

Methods and analysis Data from 150 Dutch BS patients (ages between 35 and 55, men and women) will be collected at various time points between 2 months before and up to 10 years after surgery. Neuropsychological tests, questionnaires, blood, faeces and tissue samples will be collected before, during and after surgery to measure changes in cognition, microbiota, metabolic activity and inflammation over time. A subgroup of 75 participants will undergo (f)MRI in relation to executive functioning (determined by the Stroop task), grey and white matter volumes and cerebral blood flow. Regression analyses will be used to explore associations between weight loss and outcome measures.

Ethics and dissemination This study has been approved by the medical review ethics committee CMO Region Arnhem and Nijmegen (NL63493.091.17). Research findings will be published in peer-reviewed journals and at conferences.

Trial registration number NTR7288.

\section{Strengths and limitations of this study}

For the first time in bariatric research, neuroimaging, neuropsychological tests and metabolic and histopathological parameters will be combined to investigate the effect of weight loss on brain function and structure after bariatric surgery.

- Collecting and investigating multiple metabolic parameters (obtained from blood, various tissue and microbiota) may help reveal the relationship, and underlying mechanisms, between obesity and brain function and structure.

- With a follow-up of 10 years after bariatric surgery, additional knowledge will be gathered on the longterm effects of weight loss on cognitive function.

- Only bariatric surgery patients are included in this study, so whether the results are generalisable to patients with obesity in general will require further investigation.

\section{INTRODUCTION}

For over two decades, obesity-induced diseases, such as cardiovascular disease, and type 2 diabetes mellitus (T2DM), have been one of the major healthcare challenges of today's society. ${ }^{1}$ Besides the well-known metabolic complications, obesity may lead to structural brain changes, cognitive impairment and neurodegenerative diseases. ${ }^{2-5}$ Additionally, a direct relationship exists between increased body mass and cognitive impairment. ${ }^{6-9}$ To improve and possibly reduce the amount of obesity-induced diseases, inhibit cognitive impairment and reduce neurodegenerative diseases, sustainable long-term weight loss in patients with obesity must be achieved. Non-surgical treatments for obesity, 
such as dietary restriction and physical activity, often show disappointing long-term effects, especially in patients with morbid obesity (body mass index (BMI) above $\left.40 \mathrm{~kg} / \mathrm{m}^{2}\right) .{ }^{10}{ }^{11}$ Bariatric surgery (BS) is known to a rapid and sustainable decrease in body mass. In particular, the commonly performed Roux-en-Y gastric bypass (RYGB) leads to rapid weight loss which is often accompanied by remission of T2DM, hypertension (HT) and dyslipidaemia (DL). ${ }^{12}{ }^{13}$ RYGB is a restrictive and malabsorptive (for micronutrients) surgical procedure; it excludes the main part of the stomach, the duodenum and the first part of the jejunum from the passage of food, leading to, among others, hormonal and gut microbiota changes. ${ }^{14} 15$ Gut microbiota changes after RYGB comprise increases in gut microbiota diversity, increases in relative abundance of Actinobacteria and Firmicutes phyla and decreases in relative abundance of Bacteroidetes phylum. However, effects in reported studies are quite inconsistent and further research is needed. ${ }^{1617}$

Besides weight loss and remission of comorbidities, RYGB surgery is also associated with improved cognitive functions. ${ }^{18} 19$ This may be related to multiple metabolic parameters, such as systolic blood pressure or triglyceride concentrations. ${ }^{20}$ Metabolic complications may also arise in patients with obesity due to a disturbed interaction between metabolic organs such as adipose tissue, liver and gut. This is especially a problem in mid-life (ages between 35 and 55) in which obesity has been reported to cause cognitive decline and increase risk for developing dementia. However, mechanisms involved in this organ-organ crosstalk are poorly understood. ${ }^{41-24}$ First, one proposed mechanism is the altered signalling of visceral and abdominal adipose tissue; adipose tissue acts as an independent endocrine organ releasing several hormones, proteins and cytokines, referred to as adipokines. Obesity is associated with dysfunctional white adipose tissue and therefore an imbalance in adipokines, such as increased levels of leptin and angiotensinogen, and low levels of adiponectin and omentin. ${ }^{25}{ }^{26}$ Especially, visceral adipose tissue seems to produce unfavourable adipokines associated with more metabolic complications when compared with subcutaneous adipose tissue..$^{27-30}$ Importantly, the distribution of fat tissue depots differs between sexes. Overall, men accumulate more abdominal and visceral fat than women. ${ }^{30}$ Moreover, women have a higher level of adipokines such as leptin and adiponectin. ${ }^{31} 32$ This disbalance in adipokines may induce inflammation in several organs such as the liver, gut and vascular endothelium. The latter causing atherosclerosis, ultimately leading to changes in cerebral blood flow $(\mathrm{CBF}) .^{25}$

Second, signalling between, and within other organs, such as the liver, might be altered in patients with obesity. For example, the liver secretes hepatokines, such as insulin-like growth factor 1 , selenoprotein $\mathrm{P}$, leucocyte cell-derived chemotaxin, fetuin B and hepassocin, which may indirectly affect brain function and structure. $^{33} 34$
Third, the gut microbiota composition in obese people differs from that of non-obese individuals, affecting metabolic processes, weight and obesity-related comorbidities. ${ }^{35}$ Microbiota is involved in adiposity and homeostasis but also influences energy balance via appetite and satiety signalling to the brain. Gut microbiota also affects the brain by producing (precursors of) neurotransmitters and short-chain fatty acids, or through cytokines via the immune system. ${ }^{37}{ }^{38}$ BS leads to a fast change in gut microbiota composition through changes in food intake, intestinal modifications due to the surgery itself and metabolic improvements, eventually leading to changes in gut-brain communication. ${ }^{15940}$ Hence, metabolic organs, such as liver, gut and adipose tissue and gut microbiota, may constitute new therapeutic targets. Although long-term results are not yet clear, the gut microbiota has already become a target for antiobesity treatments. ${ }^{37}$

Obesity is associated with impaired CBF, which may lead to inadequate oxygen and energy supply in the brain and eventually loss of white and grey matter integrity. ${ }^{41}$ Lower levels of $\mathrm{CBF}$ in the prefrontal cortex are associated with reduced performance on executive function and episodic memory tests. ${ }^{42}{ }^{43}$ Even in the prodromal stages of Alzheimer's disease, changes in CBF can be detected with arterial spin labelling (ASL), which may be used as a very early biomarker for neurodegenerative disorders. ${ }^{44}$ However, the technique requires further optimisation and therefore several consortia are working on the implementation of ASL perfusion MRI for clinical applications to provide images of sufficient and diagnostic utility. ${ }^{45}$

Furthermore, obesity is associated with changes in grey and white matter, which can be visualised using diffusion tensor imaging (DTI) and voxel-based morphometry analyses based on T1-weighted scans. ${ }^{46} 47$ These structural changes are especially prominent in brain regions governing reward seeking, inhibitory control and appetite. ${ }^{489}$ There are indications that rapid recovery of structural abnormalities occurs after BS, however long-term study data are lacking here. ${ }^{50} 51$

Additionally, impairment in attention span, executive function and memory are commonly reported in patients with obesity. ${ }^{18} 19$ Cognitive impairment revealed in obesity might be reversible and varies between cognitive domains, however long-term follow-up studies are scarce. The Longitudinal Assessment of Bariatric Surgery parent project is the most extensive longitudinal study to date focusing on cognitive changes in patients after BS. Investigators showed lasting improvements 3 years after surgery in the cognitive domains of attention, executive function and memory. ${ }^{19}$

\section{Rationale}

Cognitive benefits after BS are not equally exhibited across patients and cognitive domains. However, precise causes are still poorly understood, and underlying molecular mechanisms remain elusive. From the relatively short follow-up duration and small samples of BS patients in the 
studies reviewed, it is difficult to elaborate on the longterm consequences of obesity and its related diseases. In this study, underlying mechanisms of obesity-related cognitive disorders will be investigated by longitudinal studies correlating cognition to brain changes, blood serum and plasma values, and gut microbiota composition. Lastly, metabolic and histopathological parameters (at the time point of surgery) will be obtained to study whether associations or correlations exist between obesity-associated metabolic dysfunctions of particular organs and brain function and structure. To our knowledge, this is the first study in humans investigating changes in brain structure and function, and changes in adipose tissue, liver function and the gut microbiome, after BS-induced weight loss. Additionally, this is the first study in bariatric research combining neuroimaging, cognition and extensive profiling of biological markers.

The primary aim of the BARICO study (BAriatric surgery Rijnstate and Radboudumc neurolmaging and Cognition in Obesity) is to determine the long-term effect of weight loss on measures of brain function and structure after BS. The secondary aim is to provide mechanism-based rationales responsible for functional and structural decline in obese individuals. Therefore, the metabolic and inflammation status of organ biopsies will be determined together with molecular signatures via blood plasma/serum analyses. Furthermore, gut microbiota composition will be monitored over time to gain knowledge about the gut-brain axis.

This study will contribute to the development of better health campaigns, healthcare and preventives to attenuate the impact of obesity. This paper describes the design and protocol of the BARICO study.

\section{METHODS AND ANALYSIS}

\section{Study population}

Patients who have been screened and found eligible for BS based on the Fried guidelines will be asked to participate. $^{52}$ In total, 150 patients will be included in the study. Study-specific inclusion criteria are: (A) patients willing to perform neuropsychological tests, complete self-report questionnaires and sign an informed consent document; (B) ages between 35 and 55 years; and (C) patients must undergo RYGB. A laparoscopic antecolic antegastric RYGB procedure will be performed (biliopancreatic limb of $150 \mathrm{~cm}$, alimentary limb of $100 \mathrm{~cm}$ ). Exclusion criteria for this study are: (A) previous or current neurological or severe psychiatric illness; (B) pregnancy; and $(\mathrm{C})$ treatment with any antibiotics, probiotics or prebiotics 3 months before or at any point during the study (excluding preoperative prophylaxis). A subgroup of 75 patients will be included in the MRI substudy, extra inclusion criteria for this group are: (D) patients willing to undergo MRI scanning and perform tasks in the MRI scanner; and (E) right handed (more homogeneous sample and less variance). The standard exclusion criteria for the MRI subgroup include: (F) claustrophobia; (G) epilepsy; (H) pacemakers and defibrillators; (I) nerve stimulators; (J) intracranial clips; (K) infraorbital or intraocular metallic fragments; (L) cochlear implants; (M) ferromagnetic implants; (N) circumference above the MRI space capacity; and $(\mathrm{O})$ colour blindness. The study is registered at the Netherlands Trial Register (trialregister.nl: NTR7288).

\section{Study design}

At several time points (4-8 weeks preoperative; 6, 24 months; and 5, 10 years postoperative (figure 1)), a number of cognitive tests and questionnaires will be performed, and their results assessed. Furthermore, blood (after 8 hours period of fasting) and faecal matter will be collected from all patients $(n=150)$ (blood at all time points, faeces $4-8$ weeks preoperative, 6 and 24 months postoperative (figure 1)). Intraoperatively, several tissue biopsies will be collected and processed. Medical evaluation, including anthropometric measurements and information on comorbidities, will be assessed 4-8 weeks preoperative and during all postoperative time points. A schematic overview of the study is shown in figure 1. A subgroup of patients $(\mathrm{n}=75)$ will additionally receive a (functional) MRI ((f)MRI) scan 4-8 weeks preoperative and 24 months postoperative. During the whole study period (10 years), patients will be contacted by letter and via telephone at least once a year to ensure the best follow-up rate.

\section{Recruitment procedures and consent}

Patients are informed about the study by letter and telephone at least 2 weeks prior to their standard visit (4-8 weeks before RYGB surgery). During this visit, patients will individually receive more information about this study and its objectives. Afterwards, the researchers will further clarify the study and the patients can ask for additional information. If they decide to participate and fulfil the inclusion criteria, informed consents will be obtained. Although the obese population consists of more females than males, the aim is for an equal sex distribution during the recruitment period (ie, a study population consisting of $>30 \%$ men and $>30 \%$ women). ${ }^{1}$ Recruitment will take place between August 2018 and August 2020.

\section{Outcome measures}

The primary outcome measures are the neuropsychological test scores, CBF values, hippocampal volume, mean diffusivity (MD) and fractional anisotropy (FA) (representing respectively grey and white matter integrity), and blood oxygen level dependent (BOLD) responses during the Stroop task. Combining neuroimaging and neuropsychological tests will give us more information on how and whether structural brain changes are related to functional brain changes. Secondary measures comprise (histopathologically and biochemically determined) health status of the collected tissue, gut microbiota composition changes (in jejunal mucosa and faeces) and the profiling of circulating mediators in blood (plasma and serum), as 
4-8 wks pre BS

BS

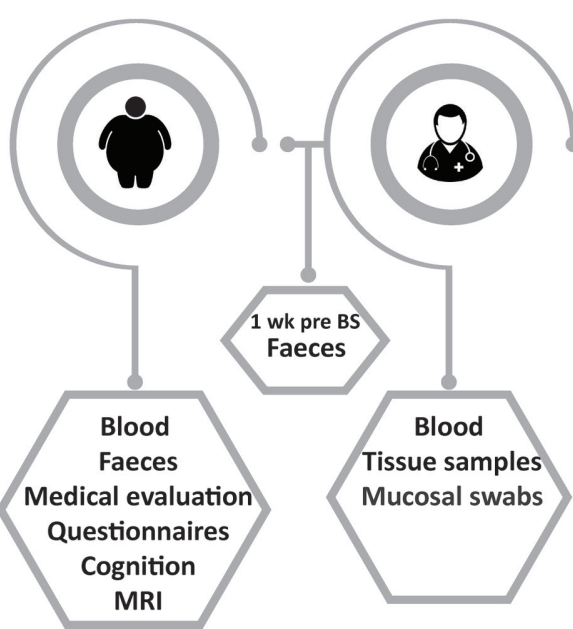

6 mo post BS

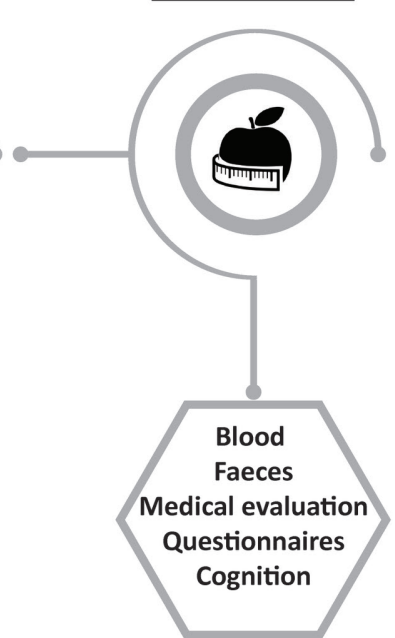

24 mo post BS

$\underline{5 \& 10 \text { yrs post BS }}$

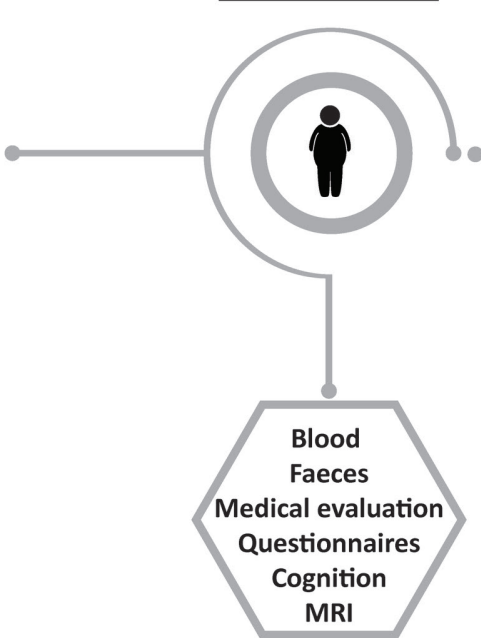

Figure 1 Overview of the study design. Blood samples are taken during a regular blood withdrawal at six time points (4-8 weeks before BS, BS, 6 months after BS, 24 months after BS, and 5 and 10 years after BS). Microbiota analyses will be performed at set time points on the faeces (collected at home by the patients) (4-8 weeks before BS, 1 week before BS, 6 months after BS, 24 months after BS) and mucosal swabs (collected during surgery). Furthermore, biopsies of liver, jejunum and adipose tissue depots (subcutaneous, mesenteric and omental) will be collected during surgery. Before surgery (4-8 weeks before BS) and at several time points after ( 6 months after BS, 24 months after BS, and 5 and 10 years after BS), a medical evaluation will take place and all patients will complete questionnaires and neuropsychological measurements to test cognitive function. A subgroup of patients ( $n=75$ ) will be examined with MRI (4-8 weeks before BS and 24 months after BS). BS, bariatric surgery.

well as lifestyle and dietary habits in relation to cognitive function and brain structure. Combining information on the pathological state of liver, gut, adipose tissue and circulating mediators from corresponding plasma/serum samples, obtained prior to and at surgery, will provide insight into organ crosstalk and allow identification of biomarker signatures for metabolic health. Differences in metabolic health of the subjects may be associated with specific signalling molecule profiles, which may be related to cognitive function.

\section{(Functional) MRI}

Subjects will be scanned in a 3T MAGNETOM Skyra MR scanner (Siemens, Healthcare Sector, Erlangen, Germany) using a 32-channel head coil. The MRI protocol included: a T1-weighted 3D magnetisation-prepared rapid gradient-echo sequence for anatomical reference and analysis (TR/TI/TE 2300/1100/3.03 ms; $8^{\circ}$ flip angle; voxel size: $1.0 \times 1.0 \times 1.0 \mathrm{~mm}$ ), a fluid-attenuated inversion recovery sequence for white matter lesion visualisation (TR/TI $5000 / 1800 \mathrm{~ms}$; voxel size: $1.0 \times 1.0 \times 1.0 \mathrm{~mm}$ ) and diffusion-weighted MRI scans using simultaneous multislice echo planar imaging for probing microstructural properties (repetition time/echo time (TR/TE) 3275/91.4 ms; voxel size: $1.9 \times 1.9 \times 1.9 \mathrm{~mm} ; 6 \times \mathrm{b}=0 \mathrm{~s} / \mathrm{mm}^{2}, 42 \times \mathrm{b}=900 \mathrm{~s} /$ $\mathrm{mm}^{2}, 83 \times \mathrm{b}=1800 \mathrm{~s} / \mathrm{mm}^{2}$ ). To allow for offline distortion correction of the images, seven more $b=0 \mathrm{~s} / \mathrm{mm}^{2}$ volumes will be acquired using the exact same sequence parameters-except for the inverted k-space read-out trajectory. An ASL sequence will be used for quantification of CBF (TR/TE 2500/12 ms; voxel size: $4.0 \times 4.0 \times 4.0 \mathrm{~mm}$ ) and a multiband, multiecho planar imaging sequence will be used to measure BOLD contrast during the Stroop task (TR/TE $1500 / 12.4,34.3,56.2 \mathrm{~ms} ; 75^{\circ}$ flip angle; voxel size: $2.5 \times 2.5 \times 2.5 \mathrm{~mm}$; field of view $210 \mathrm{~mm}$; 51 transversal slices in interleaved order). The complete scanning protocol takes $45 \mathrm{~min}$ and for both time points, the same: MR scanner, head coil and sequences will be used. Following the project MRI quality assurance is guaranteed by regular phantom measurements.

\section{Cognitive assessment}

Cognitive performance of all participants will be tested using an extensive neuropsychological test battery as detailed below. To assess general cognitive performance the Montreal Cognitive Assessment (MoCA) will be used. ${ }^{53}$ To test attentional functions, the Flexibility subtest from the Tests of Attentional Performance (2.3) will be used. ${ }^{54}$ This flexibility task focuses on shifting attention between objects. Working memory will be assessed via the Digit Span subtest from the Dutch language version of the Wechsler Adult Intelligence Scale-Fourth Edition. ${ }^{55}$ Participants will have to repeat a series of digits in forward or backward order, or sort them numerically. The Controlled Oral Word Association Test (COWAT) will be used to determine verbal fluency. ${ }^{56}$ Participants have to come up with as many words beginning with three designated letters within $60 \mathrm{~s}$ (for each letter). Episodic memory will be assessed via the immediate and delayed Story Recall subtest from the Rivermead Behavioural Memory Test. ${ }^{57}$ To control and correct for differences in premorbid intelligence between participants, verbal IQ will be estimated using the Dutch version of the National Adult Reading Test at baseline. ${ }^{58}$ The MoCA, episodic 
memory test and COWAT have parallel versions, to avoid material-specific learning effects during the repeated testing. Additionally, the tests are standardised, have been validated for use across a wide age range and have good retest reliability. Together, these tests will provide a good overview on the overall cognitive performance of the patients, including aspects of working and episodic memory, attention, verbal fluency and executive function. Also, education level will be recorded in accordance with the Dutch education system using seven categories ( 1 being the lowest level of education and 7 being the highest). ${ }^{59}$

\section{Assessment of biological measurements}

At several time points (figure 1), fasting (at least 8 hours) blood samples from the participants will be collected. As standard procedure, classical parameters, such as several vitamins (vitamin $\mathrm{B}_{12}$, vitamin $\mathrm{D}$ and folic acid) and lipids (triglycerides and cholesterol), will be measured. Special interest is taken on circulating mediators of organ crosstalk, such as cytokines, oxylipids, adipokines, hormones and inflammation markers (eg, C-reactive protein, serum amyloid A, vascular cell adhesion molecule 1, transforming growth factor beta), as well as metabolites (derived from organs or microbiota) assessed by metabolomics, such as bile acids and bioactive (short-chain) fatty acids, and other lipid species (untargeted lipidomics).

Besides blood samples, faeces will be collected (figure 1) using 'faeces collection kits for at home' in order to monitor gut microbiota changes and relate them to cognition and brain structure and function read-outs. Additionally, to gain insight into the microbiota in the intestinal mucosa, mucosal swabs will be collected during surgery within the jejunum (two places; 150 and $250 \mathrm{~cm}$ from Treitz ligament) and stomach pouch.

As metabolically active organs such as the liver and adipose tissue interact directly and indirectly with the brain, biopsies of these organs will be collected and analysed on histopathological and biochemical level. Tissue biopsies will be collected from subcutaneous, mesenteric and omental adipose tissue, liver and jejunum. Tissue biopsies from these organs will be taken to assess potential pathophysiological processes and to eventually define mechanism-based subgroups.

\section{Questionnaires}

At several time points (figure 1) standardised questionnaires on lifestyle, education, success rate of the surgery and eating habits will be assessed. Most of the questionnaires are routine practice for patients undergoing BS at the Rijnstate Hospital. Physical activity will be assessed via the Baecke questionnaire and depressive symptoms will be assessed with the Beck Depression Inventory II. ${ }^{60} 61$ To estimate the participants' food/nutrient intake and eating behaviour patients will be asked to fill out an eating diary of 2 days (a weekday and a weekend day). Quality of life will be evaluated with the Short-Form $36 .{ }^{62}$ Lastly, the results of BS will be evaluated via the Bariatric Analysis and Report Outcome System. ${ }^{63}$

More specifically, the Barratt Impulsivity Scale- $11^{64}$ and Behavioural Inhibition/Activation System ${ }^{65}$ questionnaires on impulsivity and reward sensitivity are included as reward sensitivity and impulsivity have both previously been suggested to contribute to overeating. ${ }^{66}$ Indeed, some facets of impulsivity and reward sensitivity have shown to be relevant in eating and weight regulation. ${ }^{67}$

\section{Medical evaluation}

At several time points during the study (figure 1) a medical evaluation will take place where anthropometric measurements, such as body weight, length, waist circumference and blood pressure, will be quantified. BMI will be calculated as weight divided by height in metres squared. Percentage excess weight loss (\%EWL) (defined as weight loss divided by preoperative excess weight, with excess weight defined as the weight above a normal BMI of $25 \mathrm{~kg} / \mathrm{m}^{2}$ ) will be calculated during the time points after surgery, similar to percentage total body weight loss (\% TBWL) (defined as weight loss divided by preoperative weight). The success of BS in terms of weight loss will be defined as a sustained weight loss larger than $50 \% \mathrm{EWL}$.

Furthermore, data on comorbidities like T2DM, HT and DL and associated medication will be collected before the surgery and at all time points after surgery. Comorbidities will be defined using the following criteria: for T2DM a fasting plasma glucose of $\geq 7.0 \mathrm{mmol} / \mathrm{L}$ and $\mathrm{HbA} 1 \mathrm{c} \geq 48 \mathrm{mmol} / \mathrm{mol}(\mathrm{HbAlc} \geq 6.5 \%)$ or the use of oral antidiabetic or insulin medication; for HT the use of antihypertensive drug treatment; for DL the use of statins.

\section{Data management}

Data management will be handled using Research Manager (Cloud 9 Health Solutions@), an established software package and data management tool that follows Good Clinical Practice guidelines. ${ }^{68}$ Every change in the data is recorded in a log system and can be traced. Participants will be identified only by a study-specific identification code. One researcher will keep a separate participant identification code list that matches the study-specific identifying codes with the participant's names. Documents will be maintained by the investigator in strict confidence.

\section{Sample size}

The power calculation for the neuropsychological tasks was based on the results of the Digit Span subtest performed in a comparable study population. ${ }^{19}$ With an expected standardised effect size of at least 0.3 and a correlation of 0.7 , a selected sample of 150 patients will be sufficient to reach $90 \%$ power. The power calculation for the MRI parameters is based on changes in the FA parameter studied by Zhang et al. ${ }^{51}$ With an expected standardised effect size of at least 0.03 and a correlation of 0.5 including 75 patients in the MRI group will be sufficient to reach $90 \%$ power. A significance level based 
on the sequentially rejective multiple testing procedure discussed by Bretz et al (for the neuropsychological tests $3 \%$ and for the MRI parameters $2 \%$ ) has been taken into account in the power calculation. ${ }^{69}$ The inclusion of 150 patients with a subgroup of 75 for the MRI scan has been considered adequate to answer the research questions with sufficient power.

\section{Analysis of primary outcome measures}

As a primary outcome measure, baseline levels of the imaging parameters (such as MD and FA) will be compared with the results of the neuroimaging outcome 24 months after surgery (including \% TBWL as a factor in the model). Next, the scores of the cognitive tests from five different time points will be analysed and compared with $\%$ TBWL. Every dependent variable will be modelled in a separate linear mixed model. \% TBWL will be used as a factor. Different variables, such as depression score, age and gender, will be (if appropriate) included in the model. For each model, we will decide which variables to include as a factor to reduce the amount of unexplained variation. To correct for multiple outcome measures, the sequentially rejective multiple testing procedure described in Bretz et al will be used (more information in the online supplementary material). ${ }^{69}$ Data will be analysed using SPSS (V.25 for Windows) and R (V.3.5.1 for Windows). For the cognitive tests and the imaging parameters, a p value $<0.03$ and $<0.02$, respectively, will be considered as statistically significant.

\section{Analysis of secondary outcome measures}

As secondary outcome measures, the metabolic and histopathological parameters (obtained analyses from tissues collected during surgery) will be analysed cross-sectionally to examine correlations between and among each other, and in relation to brain function and structure. Furthermore, potential mechanisms underlying the crosstalk along the gut-brain axis will be investigated by longitudinal analyses focusing on establishing correlations between brain structure/function changes and changes in circulation mediators or faecal microbiota composition. Pearson correlation analysis will be used to investigate potential correlations between variables.

\section{Data monitoring}

Every year, data monitoring and auditing will be conducted by an independent specialised monitor from the Rijnstate Hospital. Yearly, a summary of the progress will be submitted to the ethical committee and the Netherlands Trial Register (trialregister.nl: NTR7288).

\section{Patient and public involvement}

Patients and the public were not involved in the design of this study. Nevertheless, the results will be disseminated to the study participants via email, newsletters and social media platforms after the study results are published.

\section{DISCUSSION}

The BARICO study is a prospective study focusing on the effect of weight loss on cognitive function and brain structure after BS. This will be measured using sensitive neuropsychological tests covering the most important domains, (f)MRI activation during the Stroop task and several MRI techniques, such as DTI and ASL. To clarify the impact of metabolic dysfunction in obesity on brain function and structure, blood plasma and stool samples will be collected and analysed longitudinally, and biopsies of key metabolic organs will be collected during the RYGB and analysed cross-sectionally.

After BS, there have only been a limited number of long-term studies demonstrating improvement in several cognitive domains, including memory, attention and executive function. ${ }^{18}{ }^{19}$ Furthermore, it has been shown that obese individuals have lower grey and white matter volumes, and altered white matter densities, in comparison to healthy individuals with several studies showing a rapid recovery of these brain structural abnormalities after BS. ${ }^{5051}$ For instance, Tuulari et alshowed a causal link between weight loss and brain tissue recovery. ${ }^{50}$ Approximately $25 \%-30 \%$ of the patients are not expected to reach sufficient weight loss ( $\leq 50 \% \mathrm{EWL})$, and thus it will be possible to study the effect of weight loss after BS on brain function and structure.

Perhaps the strength of this study is in the long follow-up duration after surgery: 24 months for the neuroimaging parameters and 10 years for the neuropsychological tests. Furthermore, the strict inclusion criterion with respect to age range ensures a good representation of mid-life patients. Moreover, the majority of studies into BS patients are mostly composed of women, but it is equally important to account for the variation in fat tissue distribution which is caused by differences in sex. ${ }^{30}$

Another strength of this study is the combination of neuroimaging and neuropsychological tests. Alongside the analysis of metabolic and histopathological parameters (obtained in blood, organ biopsies and microbiota), meaning that the relation between multiple metabolic, neuroimaging and/or cognitive parameters can be investigated (eg, adipokines, bioactive lipids (short-chain fatty acids) and organ dysfunction) in a comprehensive way. Since RYGB influences gut-brain communication, there may be beneficial alterations in adipose tissue functions, and/or recovery of brain function and structure following BS. ${ }^{15} 70$ Longitudinal analyses of the microbiota, together with analysis of functional gut-derived metabolites in the circulation and cognitive outcomes, may allow for the identification of mediators derived from gut microflora that are relevant to cognition and the prevention of cognitive decline.

The BARICO study has the potential to be the first to demonstrate interactions between the periphery and central nervous system after weight loss in humans, in particular it will question the roles and involvement of the brain, and adipose tissue, liver and gut microbiota, after weight loss caused by BS. 
In conclusion, the BARICO study will reveal the relation and underlying mechanisms between obesity and brain function and structure. This information can be used to develop better healthcare as well as possible preventives against obesity and associated disorders.

\section{Author affiliations}

${ }^{1}$ Department of Surgery, Rijnstate Hospital, Vitalys Clinic, Arnhem, The Netherlands

${ }^{2}$ Department of Anatomy, Donders Institute for Brain, Cognition and Behaviour, Radboud University Medical Center, Nijmegen, The Netherlands

${ }^{3}$ Donders Institute for Brain, Cognition and Behaviour, Radboud University, Nijmegen, The Netherlands

${ }^{4}$ Department of Medical Psychology, Radboud University Medical Center, Nijmegen, The Netherlands

${ }^{5}$ Vincent van Gogh Institute for Psychiatry, Venray, The Netherlands

${ }^{6}$ Department of Metabolic Health Research, Netherlands Organization for Applied

Scientific Research (TNO), Leiden, The Netherlands

Contributors EOA and AJK conceived and designed the study. DV wrote the article and developed the protocol together with EOA, AJK, EJH and RK. EJH, EOA and AJK are the principal investigators and DV is the main investigator. MW, LND, IACA, EA, RK and RPCK are coinvestigators in the participating centres. All authors critically reviewed the content and approved the final manuscript.

Funding This work is supported by a grant of the Rijnstate-Radboudumc promotion fund. The histopathological and biochemical analyses will be performed in collaboration with the Netherlands Organisation for Applied Scientific Research (TNO) Metabolic Health Research (Leiden, the Netherlands) with support from TNO's Research programme Biomedical Health and the Shared Research Program GLoBAL, an initiative of Radboudumc, Rijnstate and TNO.

Competing interests Not required.

Patient consent Not required.

Ethics approval Medical review ethics committee CMO Region Arnhem and Nijmegen (NL63493.091.17).

Provenance and peer review Not commissioned; externally peer reviewed.

Open access This is an open access article distributed in accordance with the Creative Commons Attribution Non Commercial (CC BY-NC 4.0) license, which permits others to distribute, remix, adapt, build upon this work non-commercially, and license their derivative works on different terms, provided the original work is properly cited, appropriate credit is given, any changes made indicated, and the use is non-commercial. See: http://creativecommons.org/licenses/by-nc/4.0/.

\section{REFERENCES}

1. WHO. Obesity and overweight; Fact sheet: WHO, 2018.

2. Espeland MA, Erickson $\mathrm{K}$, Neiberg RH, et al. Brain and white matter hyperintensity volumes after 10 years of random assignment to lifestyle intervention. Diabetes Care 2016;39:764-71.

3. Anstey KJ, Cherbuin N, Budge M, et al. Body mass index in midlife and late-life as a risk factor for dementia: a meta-analysis of prospective studies. Obes Rev 2011;12:e426-37.

4. Emmerzaal TL, Kiliaan AJ, Gustafson DR. 2003-2013: a decade of body mass index, Alzheimer's disease, and dementia. J Alzheimers Dis 2015;43:739-55.

5. Maayan L, Hoogendoorn C, Sweat V, et al. Disinhibited eating in obese adolescents is associated with orbitofrontal volume reductions and executive dysfunction. Obesity 2011;19:1382-7.

6. Cournot M, Marquié JC, Ansiau D, et al. Relation between body mass index and cognitive function in healthy middle-aged men and women. Neurology 2006;67:1208-14.

7. Gunstad J, Lhotsky A, Wendell CR, et al. Longitudinal examination of obesity and cognitive function: results from the Baltimore longitudinal study of aging. Neuroepidemiology 2010;34:222-9.

8. Prickett C, Brennan L, Stolwyk R. Examining the relationship between obesity and cognitive function: a systematic literature review. Obes Res Clin Pract 2015;9:93-113.

9. Bastard JP, Maachi M, Lagathu C, et al. Recent advances in the relationship between obesity, inflammation, and insulin resistance. Eur Cytokine Netw 2006;17:4-12.
10. Gloy VL, Briel M, Bhatt DL, et al. Bariatric surgery versus nonsurgical treatment for obesity: a systematic review and meta-analysis of randomised controlled trials. BMJ 2013;347:f5934.

11. Europe W. Body mass index - BMI. 2018 http://www.euro.who.int/en/ health-topics/disease-prevention/nutrition/a-healthy-lifestyle/bodymass-index-bmi

12. Carranza-Leon BG, Puzziferri N, Adams-Huet B, et al. Metabolic response 4 years after gastric bypass in a complete cohort with type 2 diabetes mellitus. Diabetes Res Clin Pract 2018;137:224-30.

13. Dogan K, Betzel B, Homan J, et al. Long-term effects of laparoscopic Roux-en-Y gastric bypass on diabetes mellitus, hypertension and dyslipidaemia in morbidly obese patients. Obes Surg 2014;24:1835-42.

14. Kim KS, Sandoval DA. Endocrine function after bariatric surgery. Compr Physiol 2017;7:783-98.

15. Ballsmider LA, Vaughn AC, David M, et al. Sleeve gastrectomy and Roux-en-Y gastric bypass alter the gut-brain communication. Neural Plast 2015;2015:1-9.

16. Murphy R, Tsai $P$, Jüllig $M$, et al. Differential changes in gut microbiota after gastric bypass and sleeve gastrectomy bariatric surgery vary according to diabetes remission. Obes Surg 2017;27:917-25.

17. Zhang $\mathrm{H}$, DiBaise JK, Zuccolo $\mathrm{A}$, et al. Human gut microbiota in obesity and after gastric bypass. Proc Natl Acad Sci U S A 2009;106:2365-70

18. Handley JD, Williams DM, Caplin S, et al. Changes in cognitive function following bariatric surgery: a systematic review. Obes Surg 2016;26:2530-7.

19. Alosco ML, Galioto R, Spitznagel MB, et al. Cognitive function after bariatric surgery: evidence for improvement 3 years after surgery. Am J Surg 2014;207:870-6.

20. Tuulari JJ. Effects of obesity and weight loss following bariatric surgery on brain function: Structural Integrity and Metabolism, 2015.

21. Kivipelto M, Ngandu T, Fratiglioni L, et al. Obesity and vascular risk factors at midlife and the risk of dementia and Alzheimer disease. Arch Neurol 2005;62:1556-60.

22. Whitmer RA, Gustafson DR, Barrett-Connor E, et al. Central obesity and increased risk of dementia more than three decades later. Neurology 2008;71:1057-64.

23. Whitmer RA, Gunderson EP, Barrett-Connor E, et al. Obesity in middle age and future risk of dementia: a 27 year longitudinal population based study. BMJ 2005;330:1360.

24. Whitmer RA, Gunderson EP, Quesenberry CP, et al. Body mass index in midlife and risk of Alzheimer disease and vascular dementia. Curr Alzheimer Res 2007;4:103-9.

25. Arnoldussen IA, Kiliaan AJ, Gustafson DR. Obesity and dementia: adipokines interact with the brain. Eur Neuropsychopharmacol 2014;24:1982-99.

26. Jaganathan R, Ravindran R, Dhanasekaran S. Emerging role of adipocytokines in type 2 diabetes as mediators of insulin resistance and cardiovascular disease. Can J Diabetes 2018;42:446-56.

27. Ahima RS, Flier JS. Adipose tissue as an endocrine organ. Trends Endocrinol Metab 2000;11:327-32.

28. Arner P. Not all fat is alike. Lancet 1998;351:1301-2.

29. Foster MT, Pagliassotti MJ. Metabolic alterations following visceral fat removal and expansion: Beyond anatomic location. Adipocyte 2012;1:192-9.

30. Lee MJ, Wu Y, Fried SK. Adipose tissue heterogeneity: implication of depot differences in adipose tissue for obesity complications. Mol Aspects Med 2013;34:1-11.

31. Considine RV, Sinha MK, Heiman ML, et al. Serum immunoreactiveleptin concentrations in normal-weight and obese humans. $N$ Engl $J$ Med 1996;334:292-5.

32. Cnop M, Havel PJ, Utzschneider KM, et al. Relationship of adiponectin to body fat distribution, insulin sensitivity and plasma lipoproteins: evidence for independent roles of age and sex. Diabetologia 2003;46:459-69.

33. Meex RCR, Watt MJ. Hepatokines: linking nonalcoholic fatty liver disease and insulin resistance. Nat Rev Endocrinol 2017;13:509-20.

34. Stefan N, Häring HU. The role of hepatokines in metabolism. Nat Rev Endocrinol 2013;9:144-52.

35. Tilg $\mathrm{H}$, Kaser A. Gut microbiome, obesity, and metabolic dysfunction. $J$ Clin Invest 2011;121:2126-32.

36. Tremaroli V, Bäckhed F. Functional interactions between the gut microbiota and host metabolism. Nature 2012;489:242-9.

37. Torres-Fuentes C, Schellekens H, Dinan TG, et al. The microbiotagut-brain axis in obesity. Lancet Gastroenterol Hepatol 2017;2:747-56.

38. Wang HX, Wang YP. Gut Microbiota-brain Axis. Chin Med J 2016;129:2373-80. 
39. Aron-Wisnewsky J, Doré J, Clement K. The importance of the gut microbiota after bariatric surgery. Nat Rev Gastroenterol Hepatol 2012;9:590-8.

40. Peat CM, Kleiman SC, Bulik CM, et al. The Intestinal microbiome in bariatric surgery patients. Eur Eat Disord Rev 2015;23:496-503.

41. Cipolla MJ. Chapter 5: Control of Cerebral Blood Flow. The cerebral circulation. San Rafael (CA): Integrated Systems Physiology. From Molecule to Function, 2009:29-36.

42. Willeumier KC, Taylor DV, Amen DG. Elevated BMI is associated with decreased blood flow in the prefrontal cortex using SPECT imaging in healthy adults. Obesity 2011;19:1095-7.

43. Alosco ML, Spitznagel MB, Raz N, et al. Obesity interacts with cerebral hypoperfusion to exacerbate cognitive impairment in older adults with heart failure. Cerebrovasc Dis Extra 2012;2:88-98.

44. Wierenga CE, Hays CC, Zlatar ZZ. Cerebral blood flow measured by arterial spin labeling MRI as a preclinical marker of Alzheimer's disease. J Alzheimers Dis 2014;42:S411-9.

45. Alsop DC, Detre JA, Golay X, et al. Recommended implementation of arterial spin-labeled perfusion mri for clinical applications: a consensus of the ISMRM perfusion study group and the European consortium for ASL in dementia. Magn Reson Med 2015;73:102-16.

46. Kullmann S, Callaghan MF, Heni M, et al. Specific white matter tissue microstructure changes associated with obesity. Neuroimage 2016;125:36-44.

47. Debette $S$, Wolf $C$, Lambert JC, et al. Abdominal obesity and lower gray matter volume: a Mendelian randomization study. Neurobiol Aging 2014;35:378-86.

48. Karlsson HK, Tuulari JJ, Hirvonen $\mathrm{J}$, et al. Obesity is associated with white matter atrophy: a combined diffusion tensor imaging and voxel-based morphometric study. Obesity 2013;21:2530-7.

49. Arnoldussen IAC, Wiesmann M, Pelgrim CE, et al. Butyrate restores HFD-induced adaptations in brain function and metabolism in midadult obese mice. Int J Obes 2017;41:935-44.

50. Tuulari JJ, Karlsson HK, Antikainen O, et al. Bariatric surgery induces white and grey matter density recovery in the morbidly obese: a voxel-based morphometric study. Hum Brain Mapp 2016;37:3745-56.

51. Zhang Y, Ji G, Xu M, et al. Recovery of brain structural abnormalities in morbidly obese patients after bariatric surgery. Int $J$ Obes 2016;40:1558-65.

52. Fried M, Hainer V, Basdevant A, et al. Interdisciplinary European guidelines on surgery of severe obesity. Obes Facts 2008;1:52-9.

53. Nasreddine ZS, Phillips NA, Bédirian V, et al. The montreal cognitive assessment, moca: a brief screening tool for mild cognitive impairment. J Am Geriatr Soc 2005;53:695-9.
54. Zimmerman P, Fimm B. Test for Attentional Performance (TAP), manual. Würselen, Germany: Psytest, 1994.

55. Wechsler D. Wechsler adult intelligence scale-. Fourth Edition. San Antonio, TX: NCS Pearson, 2008:498. (WAIS-IV).

56. Schmand B, Groenink SC, den Dungen M. Letterfluency: psychometrische eigenschappen en nederlandse normen. Tijdschr Gerontol Geriatr 2008;39:64-74

57. Wilson B, Cockburn J, Baddeley A. Rivermead behavioural memory test. London: Thames Valley Test Company, 1985.

58. Schmand B, Bakker D, Saan R, et al. The dutch reading test for adults: a measure of premorbid intelligence level]. Tijdschr Gerontol Geriatr 1991;22:15-19.

59. Verhage F. Intelligentie en leeftijd: onderzoek bij nederlanders van twaalf tot zevenenzeventig jaar. Assen: Van Gorcum, 1964.

60. Baecke JA, Burema J, Frijters JE. A short questionnaire for the measurement of habitual physical activity in epidemiological studies. Am J Clin Nutr 1982;36:936-42.

61. Beck AT, Ward $\mathrm{CH}$, Mendelson M, et al. An inventory for measuring depression. Arch Gen Psychiatry 1961;4:561-71.

62. Ware JE, Sherbourne CD. The MOS 36 -item short-form health survey (SF-36). I. Conceptual framework and item selection. Med Care 1992;30:473-83.

63. Oria HE, Moorehead MK. Bariatric analysis and reporting outcome system (BAROS). Obes Surg 1998;8:487-99.

64. Patton JH, Stanford MS, Barratt ES. Factor structure of the barratt impulsiveness scale. J Clin Psychol 1995;51:768-74.

65. Carver CS, White TL. Behavioral inhibition, behavioral activation, and affective responses to impending reward and punishment: the BIS/ BAS Scales. J Pers Soc Psychol 1994;67:319-33.

66. Michaud A, Vainik U, Garcia-Garcia I, et al. Overlapping neural endophenotypes in addiction and obesity. Front Endocrinol $2017 ; 8: 127$

67. Meule A, Hofmann J, Weghuber D, et al. Impulsivity, perceived self-regulatory success in dieting, and body mass in children and adolescents: a moderated mediation model. Appetite 2016;107:15-20.

68. ICH. ICH harmonised tripartite guideline for good clinical practice: Brookwood Medical Publications Ltd, 1996.

69. Bretz F, Maurer W, Brannath W, et al. A graphical approach to sequentially rejective multiple test procedures. Stat Med 2009;28:586-604.

70. Hoffstedt J, Andersson DP, Eriksson Hogling D, et al. Long-term protective changes in adipose tissue after gastric bypass. Diabetes Care 2017:40:77-84 